\title{
Breites Anwendungsgebiet für Antikörper in der mCRC-Zweitlinientherapie
}

\author{
Die Phase-III-Studie RAISE führte zur Zulassung von Ramucirumab in \\ Kombination mit FOLFIRI beim metastasierten kolorektalen Karzinom (mCRC) \\ nach Versagen einer ersten Therapie mit Bevacizumab, Oxaliplatin und einem \\ Fluoropyrimidin. Welche Subgruppen profitieren?
}

\begin{abstract}
Ramucirumab ist ein voll humanisierRter Antikörper gegen VEGFR-2 (,vascular endothelial growth factor receptor-2"). Die Addition von Ramucirumab zu FOLFIRI hatte in der Second-Line gegenüber Placebo/FOLFIRI das Gesamtüberleben (OS; Hazard Ratio [HR] 0,844; $\mathrm{p}=0,0219)$ und das progressionsfreie Überleben (PFS; HR 0,793; p < 0,0005) signifikant verlängert. In der vorab geplante Subgruppenanalyse wurden die Faktoren KRAS-Mutationsstatus (Wildtyp [WT] vs. Mutation [Mut]), Alter (<65 vs. $\geq 65$ Jahre) und Zeit bis zum Progress
\end{abstract}

TTP führte die Addition von Ramucirumab zu FOLFIRI zu einem OS-Vorteil $\left(\mathrm{p}_{\text {Interaktion }}=0,9434\right)$. Eine TTP $<6$ Monate war aber insgesamt mit einem kürzeren OS assoziiert. Die Toxizität war in den Subgruppen vergleichbar.

(TTP) nach der Erstlinientherapie ( $<6$ vs. $\geq 6$ Monate) untersucht. Sowohl Patienten mit KRAS-WT-Tumoren als auch jene mit KRAS-Mut-Tumoren profitierten von Ramucirumab ( $\mathrm{p}_{\text {Interaktion }}=0,526$ ). Das mediane OS war bei Patienten mit KRAS-WT jedoch signifikant besser (14,4 vs. 11,9 Monate; HR 0,82; $\mathrm{p}=0,049)$ als bei Patienten mit KRAS-MUT (12,7 vs. 11,3 Monate; HR 0,89; $p=0,263$ ). Jüngere und ältere Patienten profitierten gleichermaßen von der Antikörpertherapie $\left(\mathrm{p}_{\text {Interaktion }}=0,9521\right)$. Bei Patienten mit längerer sowie Patieten mit kürzerer

Fazit: Die Analyse belegt eine vergleichbare Wirksamkeit und Sicherheit unabhängig von KRAS-Status, Alter oder TTP. Die Forscher halten Ramucirumab/ FOLFIRI daher für eine wichtige und breit anwendbare Option in der Zweitlinientherapie des mCRC. Friederike Klein

Obermannová R et al. Subgroup analysis in RAISE: a randomized, double-blind phase III study of irinotecan, folinic acid, and 5-fluorouracil (FOLFIRI) plus ramucirumab or placebo in patients with metastatic colorectal carcinoma progression. Ann Oncol. 2016;27(11):2082-90.

\section{Objektive Ansprechmuster erklären Vorteil durch Anti-EGFR-Therapie beim mCRC}

Sollte man beim metastasierten kolorektalen Karzinom (mCRC) Anti-EGFRoder Anti-VEGF-Therapien zusätzlich zur Standardchemotherapie einsetzen? Die Frage scheint hinsichtlich des Gesamtüberlebens geklärt.

In der FIRE-3-Studie wurde FOLFIRI in der First-line-Therapie des mCRC mit dem EGFR(„epidermal growth factor receptor")-Antikörper Cetuximab oder dem gegen VEGF (,vascular endothelial growth factor") gerichteten Antikörper
Bevacizumab kombiniert. In der finalen Analyse der Patienten mit RAS-Wildtyp $(\mathrm{n}=400)$ war das mediane Gesamtüberleben unter FOLFIRI/Cetuximab länger als unter FOLFIRI/Bevacizumab (33,1 vs. 25,0 Monate; Hazard Ratio [HR] 0,70,

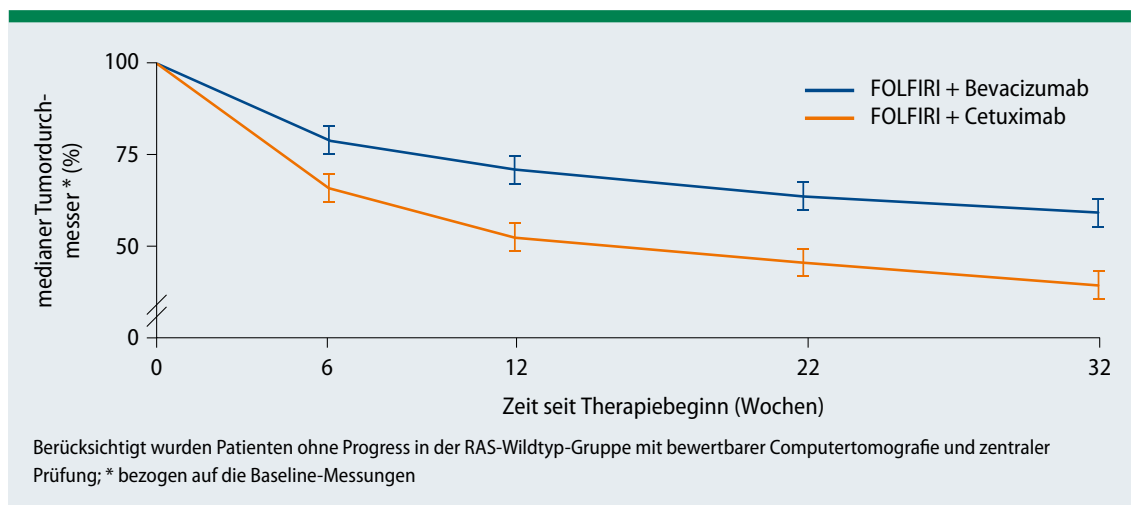

Abb. 1: Unter der Cetuximab-Addition schrumpfte der Tumor stärker.

95\%-Konfidenzintervall $\quad 0,54-0,90$; $\mathrm{p}=0,0059$ ). Ansprechen und progressionsfreies Überleben waren vergleichbar. Die Post-hoc-Analyse der zentralen radiologischen Begutachtung bei Patienten ohne RAS-Mutation betraf Aspekte der Tumordynamik (frühes Tumorschrumpfen, Ansprechtiefe, Ansprechdauer und Zeit bis zum Ansprechen). Es zeigte sich, dass Patienten im Anti-EGFR-Arm häufiger objektiv angesprochen hatten $(72,0$ vs. $56,1 \% ; p=0,0029)$, öfter ein frühes Schrumpfen des Tumors (68,2 vs. 49,1\%; $\mathrm{p}=0,0005)$ und ein median tieferes Ansprechen zeigten $(-48,9$ vs. $-32,3 \%$; $\mathrm{p}<0,0001)$. Zudem nahm der Tumordurchmesser bei Patienten ohne Progress unter Cetuximab stärker ab (Abb. 1).

Fazit: Die Post-hoc-Analyse weist auf prognostisch möglicherweise wichtige Aspekte der Dynamik des Tumoransprechens hin. So scheinen häufigeres frühes Tumorschrumpfen und größere Tiefe des Ansprechens wichtig. Friederike Klein

Stintzing $S$ et al. FOLFIRI plus cetuximab versus FOLFIRI plus bevacizumab for metastatic colorectal cancer (FIRE-3): a post-hoc analysis of tumour dynamics in the final RAS wild-type subgroup of this randomised open-label phase 3 trial. Lancet Oncol. 2016;17(10):1426-34. 